\title{
ERRATUM
}

\section{Vorticity generation and conservation for two-dimensional interfaces and boundaries - ERRATUM}

\author{
M. Brøns, M. C. Thompson, T. Leweke and K. Hourigan \\ doi:10.1017/jfm.2014.520, Published online by Cambridge University Press, \\ 7 October 2014
}

We identify an incorrect term in the expression for the rate of change of circulation of a material volume including an interface between two fluids which appears in Brøns et al. (J. Fluid Mech., vol. 758, 2014, pp. 63-93).

Brøns et al. (2014) have analysed the vorticity generation from two-dimensional interfaces as well as the vorticity flux across such interfaces. In the expressions (2.18) for the vorticity source at the interface and (2.19) for the rate of change of circulation $\mathrm{d} \Gamma / \mathrm{d} t$ of a material volume $A$ which includes both fluids, a term

$$
\int_{a}^{b} \kappa \llbracket(\boldsymbol{u} \cdot \hat{\boldsymbol{n}})(\boldsymbol{u} \cdot \hat{\boldsymbol{t}}) \rrbracket \mathrm{d} s
$$

occurs. The integral is taken along the part of the interface which is inside $A, \kappa$ is the curvature of the interface, $\boldsymbol{u}$ is the fluid velocity, $\hat{\boldsymbol{n}}$ and $\hat{\boldsymbol{t}}$ are the normal and tangential vectors of the interface, respectively, and $\llbracket q \rrbracket=q_{2}-q_{1}$ denotes the jump of a quantity $q$ across the interface.

In the derivation it is implicitly assumed that

$$
\frac{\mathrm{d}}{\mathrm{d} t} \int_{a}^{b} \gamma \mathrm{d} s=\int_{a}^{b} \frac{\mathrm{d} \gamma}{\mathrm{d} t} \mathrm{~d} s
$$

where $\gamma=\llbracket \boldsymbol{u} \cdot \hat{\boldsymbol{t}} \rrbracket$ is the strength of the vortex sheet at the interface. Terrington, Hourigan \& Thompson (2020) point out that this is valid only if the interface is stationary, and in that case (1) is zero. An expression for $\mathrm{d} \Gamma / \mathrm{d} t$ which is valid for arbitrary unsteady interfaces is derived by Terrington et al. (2020), equation (2.22), and no term like (1) occurs there. Hence, the conclusion drawn by Brøns et al. (2014), that normal motion of a curved interface is a source of vorticity given by (1), is not correct. In $\S 3$ of Brøns et al. (2014), several examples are considered. Except for one, all have a stationary interface, and here the assumption (2) leads to correct results. For the case of a solid cylinder with time-varying radius $R$ and angular velocity $\Omega$ discussed in $\S 3.2 .1$ the analysis is also correct, as (2) is not used. To compute the vorticity flux $\sigma_{1}$ into the fluid, the general expression valid for a no-slip boundary, equation (2.24), is employed:

$$
\sigma_{1}=\frac{\mathrm{d}}{\mathrm{d} t}(\boldsymbol{U} \cdot \hat{\boldsymbol{t}})+\frac{1}{\rho_{1}} \frac{\partial p_{1}}{\partial s}+\kappa(\boldsymbol{U} \cdot \hat{\boldsymbol{n}})(\boldsymbol{U} \cdot \hat{\boldsymbol{t}})-\frac{1}{2} \frac{\partial}{\partial s}(\boldsymbol{U} \cdot \hat{\boldsymbol{n}})^{2} .
$$


896 E1-2

M. Brøns, M. C. Thompson, T. Leweke and K. Hourigan

For the expanding cylinder

$$
\frac{\mathrm{d}}{\mathrm{d} t}(\boldsymbol{U} \cdot \hat{\boldsymbol{t}})=-\frac{\mathrm{d}}{\mathrm{d} t}(\Omega R), \quad \kappa(\boldsymbol{U} \cdot \hat{\boldsymbol{n}})(\boldsymbol{U} \cdot \hat{\boldsymbol{t}})=-\frac{1}{R} \frac{\mathrm{d}}{\mathrm{d} t}(\Omega R)
$$

are both in general non-zero, which after a simplification yields (3.6) in Brøns et al. (2014),

$$
\sigma_{1}=-\frac{1}{R} \frac{\mathrm{d}}{\mathrm{d} t}\left(\Omega R^{2}\right)
$$

The total flux of vorticity into the fluid is

$$
\int_{0}^{2 \pi R} \sigma_{1} \mathrm{~d} s=-2 \pi \frac{\mathrm{d}}{\mathrm{d} t}\left(\Omega R^{2}\right) .
$$

This is balanced by the rate of change of circulation of the solid cylinder,

$$
\frac{\mathrm{d} \Gamma}{\mathrm{d} t}=\frac{\mathrm{d}}{\mathrm{d} t} \int_{\text {cylinder }} \omega \mathrm{d} A=2 \pi \frac{\mathrm{d}}{\mathrm{d} t}\left(\Omega R^{2}\right) .
$$

Hence, the normal motion of the curved interface is not a source of vorticity as the total circulation is constant. However, it contributes to the flux of vorticity between the solid cylinder and the fluid.

Finally, we note that a term $\kappa \boldsymbol{u} \cdot \hat{\boldsymbol{n}}$ has inadvertently been omitted by Brøns et al. (2014) in the discussion of the role of the pressure boundary condition at fluid interfaces. This is pointed out by Terrington et al. (2020). Corrected expressions are for (2.15)

$$
\llbracket p \rrbracket=-2 \llbracket \mu\left(\frac{\partial}{\partial s}(\boldsymbol{u} \cdot \hat{\boldsymbol{t}})+\kappa \boldsymbol{u} \cdot \hat{\boldsymbol{n}}\right) \rrbracket-T \kappa
$$

- which is identical to equation (2.29) in Terrington et al. (2020) - for (2.17)

$$
\frac{\partial}{\partial s} \llbracket \frac{p}{\rho} \rrbracket=-\frac{2}{\rho_{2}} \llbracket \mu\left(\frac{\partial^{2}}{\partial s^{2}}(\boldsymbol{u} \cdot \hat{\boldsymbol{t}})+\frac{\partial}{\partial s}(\kappa \boldsymbol{u} \cdot \hat{\boldsymbol{n}})\right) \rrbracket-\frac{1}{\rho_{2}} \frac{\partial}{\partial s}(T \kappa)+\llbracket \frac{1}{\rho} \rrbracket \frac{\partial p_{1}}{\partial s}
$$

and for (2.22)

$$
\frac{\partial}{\partial s} \llbracket \frac{p}{\rho} \rrbracket=-2 \frac{\llbracket \mu \rrbracket}{\rho_{2}}\left(\frac{\partial^{2}}{\partial s^{2}}(\boldsymbol{u} \cdot \hat{\boldsymbol{t}})+\frac{\partial}{\partial s}(\kappa \boldsymbol{u} \cdot \hat{\boldsymbol{n}})\right)-\frac{1}{\rho_{2}} \frac{\partial}{\partial s}(T \kappa)+\llbracket \frac{1}{\rho} \rrbracket \frac{\partial p_{1}}{\partial s} .
$$

The latter is identical to (2.31) in Terrington et al. (2020).

\section{Acknowledgements}

Fruitful discussions with S. J. Terrington are gratefully acknowledged.

\section{REFERENCES}

Brøns, M., Thompson, M. C., Leweke, T. \& Hourigan, K. 2014 Vorticity generation and conservation for two-dimensional interfaces and boundaries. J. Fluid Mech. 758, 63-93.

Terrington, S. J., Hourigan, K. \& Thompson, M. C. 2020 The generation and conservation of vorticity: deforming interfaces and boundaries in two-dimensional flows. J. Fluid Mech. 890, A5. 